\title{
La agenda ciudadana y la percepción del desempeño institucional: el caso de Castilla y León (2015-2019)
}

\author{
Óliver Soto Sainz \\ INSTITUTO COMPLUTENSE DE CIENCIA DE LA ADMINISTRACIÓN (UCM), MADRID, ESPAÑA \\ osoto@ucm.es
}

Eva Gracia Vega

IUVENTIA SOCIDAD COOPERATIVA

eva@iuventia.es

\begin{abstract}
Gema Sánchez Medero
INSTITUTO COMPLUTENSE DE CIENCIA DE LA ADMINISTRACIÓN (UCM), MADRID, ESPAÑA gsmedero@cps.ucm.es
\end{abstract}

Resumen: El proceso de conformación de la agenda política se ha descrito como una interacción entre las agendas de los medios de comunicación y la agenda denominada "ciudadana". En todo este proceso se ha estudiado en qué medida se imponen unas $u$ otras agendas a la hora de conformarse la agenda pública. No obstante, cuando atendemos a los niveles infraestatales, observamos que ciudadanos y medios incorporan como parte de sus agendas cuestiones referenciales que no tienen un correlato directo con su entorno.

En este artículo estudiamos en qué medida, para el caso de Castilla y León, la agenda ciudadana está al margen de las agendas mediática y política de su región, conformando una situación de disgregación y distanciamiento que tiene un efecto directo en el desempeño percibido de su comunidad autónoma. Para ello empleamos una inspección cualitativa de encuestas y la técnica del grupo de discusión realizada en varias localidades intentando captar los discursos sociales de la población castellanoleonesa.

Palabras clave: Percepción de los problemas, política autonómica, Castilla y León, elecciones.

Abstract: The process of shaping the political agenda has been described as an interaction between the agendas of the media and the so called citizen agenda. Throughout this process, it has been studied to what extent one or other agenda imposes itselft when it comes to shaping the political and public agenda. However, when we attend the infrastate levels, we observe that citizens and media incorporate referential issues that do not have a direct correlation with their environment as part of their agendas.

In this article we study to what extent, in the case of Castilla y León, the agenda of citizen are outside the media and political agendas of their region, forming a situation of disintegration and distancing that has a direct effect on the perceived performance of their Autonomous Community. For this we use a qualitative inspection of surveys and the discussion group technique carried out in various municipalities trying to capture the social discourses of the Castilian-Leonese population.

Keywords: Problem perception, regional policy, Castilla y León, elections. 


\section{Introducción}

obre el proceso de conformación de la agenda política se ha escrito mucho (Foote y Hard, 1957; McCombs y Shaw, 1972; McCombs, 2006; Downs, 1972; Cobb y Elder, 1984; Dearing y Rogers, 1988 y 1996; Bouza, 2012), y en la mayoría de las ocasiones éste ha sido descrito como una interacción entre la agenda de los medios de comunicación y la agenda denominada "ciudadana". No obstante, la formación de la agenda se entiende como el proceso a través del cual ciertos problemas o cuestiones llegan a llamar la atención del gobierno como posibles asuntos de la política pública (Cobb y Elder, 1984: 67). La cuestión es saber cuál de las dos agendas, la de los medios de comunicación o la ciudadana, tiene mayor capacidad de influencia a la hora de conformar la agenda pública, y a través de qué mecanismos un tema se torna problemático y obliga a las autoridades públicas a intervenir y hacer algo (Roth, 2006: 57).

No obstante, las cuestiones de agenda suelen plantearse siempre sin tener en cuenta los múltiples niveles de gobierno, lo que lleva a asumir una traslación inmediata independiente del contexto. La agenda política nacional, ¿ condiciona en alguna medida las agendas autonómicas y locales? ¿De qué manera se superponen estas agendas diferentes? De hecho, cabe plantearse si las agendas ciudadanas son siempre un fiel reflejo de los problemas que perciben los ciudadanos, o si éstos asumen como asuntos de interés en sus propias agendas personales temas o cuestiones que no obedecen a sus situaciones personales (Anton et al., 2007). En este artículo estudiamos en qué medida, para el caso de Castilla y León, las agendas internas ciudadanas están al margen de las agendas mediática y política de la región y el efecto directo de esta situación en la percepción del desempeño político regional por parte de la ciudadanía castellanoleonesa.

Por este motivo, en este artículo se plantea como objetivo averiguar en qué medida las agendas internas ciudadanas en Castilla y León se encuentran al margen de la agenda de su región, conformando una situación de disgregación y distanciamiento que tiene un efecto directo sobre los ciudadanos en la percepción hacia su comunidad autónoma. Así estudiaremos la agenda ciudadana tal y como se refleja en las encuestas realizadas en Castilla y León para, después, contrastarla mediante el trabajo de campo cualitativo con la percepción ciudadana de cómo se cristaliza dicha agenda. Con ello intentaremos ver la interacción de ambas agendas, la ciudadana y la política, así como los cruces que se producen entre las agendas nacionales y las autonómicas.

Para acometer este trabajo, en primer lugar, repasaremos la bibliografía que existe sobre la materia de la conformación de la agenda, prestando especial atención a los estudios realizados a nivel infraestatal. En segundo lugar, mostraremos nuestro 
marco metodológico y el tipo de estudio acometido, marcando los criterios de selección empleados, así como la hipótesis de partida de este trabajo. En tercer lugar, estudiaremos la percepción que tienen los ciudadanos de Castilla y León sobre las cuestiones más relevantes en su Comunidad Autónoma, tanto desde un punto de vista cuantitativo como cualitativo. Por último, ofreceremos una serie de conclusiones.

\section{La conformación de la agenda pública como parte del proceso democrático de respuesta a las demandas}

La democracia es el sistema político en el que se toman las decisiones por el criterio de la mayoría, de forma libre y con igual peso de los votos, respetando el derecho de la minoría a oponerse y a poder convertirse alguna vez en mayoría por métodos pacíficos (Sartori, 2005: 54; Soto, 2015: 37). No obstante, la cuestión de la democracia no es exclusivamente de cualidad (se es o no democracia), sino también de grado. Así, Dahl (1990: 13) señala que "el gobierno democrático se caracteriza fundamentalmente por su continua actitud para responder a las preferencias de sus ciudadanos, sin establecer diferencias políticas entre ellos". Esta capacidad para responder a las demandas de la ciudadanía nos remite a dos ideas: de un lado, la rendición de cuentas ante sus ciudadanos de cara a las elecciones; del otro, la opinión pública como mecanismo entre elecciones para poder medir las preferencias de los ciudadanos como uno de los baremos para calibrar la actitud de los gobiernos ante las demandas ciudadanas. Por tanto, para ver en qué medida un sistema democrático está abierto a las demandas necesitamos conocer dichas demandas y ver cómo se canalizan efectivamente. Tal y como señalan Cobb y Elder (1984) y Roth (2006), la conformación de la agenda constituye el primer paso en el ciclo de generación de las políticas públicas.

\subsection{Agenda personal, agenda pública, agenda de los medios y agenda política}

Llegados a este punto, cabe distinguir entre cuatro conceptos distintos de agenda. Por un lado, tenemos la agenda personal, que es el conjunto de problemas que más preocupan de una forma directa e individual a los ciudadanos (Bouza y Rodríguez, 2004). La agenda pública (public agenda) "es el grado o jerarquía de importancia que da el público a determinados temas durante un período de tiempo" (Dearing y Rogers, 1996: 40). Por otra parte, la agenda de los medios (media agenda) es el conjunto de noticias que aparecen sobre un determinado tema a lo largo de un espacio de tiempo (Rodríguez, 2001: 28). La agenda política (policy agenda) "mide el tipo de acciones políticas que se toman por parte de los gobiernos, parlamentos u órganos políticos" (Rodríguez, 2001: 39) y que a su vez influirán en la agenda pública y la agenda de los 
medios. De esta forma, las tres agendas constituyen un sistema en el que interactúan y se influyen mutuamente conformando los temas de mayor relevancia para el país.

Ahora bien, dependiendo de la postura que se adopte así se considerará que sea una u otra agenda la que prime en la fijación de los temas que serán objeto de medidas políticas. Así, determinados autores (Gittlin, 1979) sostienen que son los medios de comunicación quienes ejercen una poderosa influencia sobre el público que los sintoniza. Desde esta perspectiva, los mass media serían entonces los responsables de la aparición de determinados temas en la agenda pública, imponiéndolos a partir de los contenidos informativos que ofrecen. En la postura contraria, otros autores (Katz y Larzarsfeld, 1979) apuntan que la capacidad de influencia de los medios de comunicación en los receptores de información es limitada. Así, el público de los mass media procesa selectivamente la información que recibe, conforma sus opiniones en grupos reducidos (donde los liderazgos puntuales son clave) y toma sus decisiones en función de pautas culturales locales. Desde esta perspectiva, los medios de comunicación no son quienes imponen la agenda, sino que esto descansa en los ciudadanos. Tamayo y Carrillo (2005) presentan una visión más matizada para el caso español, estableciendo que será en función de los temas como veremos a una u otra agenda imponerse, en un complejo juego entrecruzado de relación.

Por otra parte, las teorías de la agenda se han preguntado por el proceso mediante el cual los temas entran en la agenda pública. Dicha cuestión ha de verse íntimamente ligada a la anterior: el proceso de entrada explica en parte la mayor importancia de las agendas política, mediática y personal para conformar la agenda pública. Siguiendo a Foote y Hart (1953: 310-317), podemos identificar cinco fases en la formación de la opinión pública:

1. Fase del problema: una situación concreta es percibida como problemática por una persona o por un grupo determinado. Con el paso del tiempo dicha percepción se generaliza entre la población o una parte de ésta. Hacia el final de la primera fase, el problema toma forma, se resume en una serie de claves y la gente implicada tiene una idea vaga de lo que quiere.

2. Fase de propuesta: comienzan a aflorar manifestaciones colectivas del problema, y de esta manera, se formulan una o varias posibles respuestas al problema.

3. Fase política: en esta fase se discuten de forma pública las propuestas alternativas que han aflorado en la fase precedente. Aquí los actores implicados que perciben que la situación afecta a sus intereses buscan apoyos (de otros lobbies, de medios de comunicación) para hacer llegar sus reivindicaciones a los grupos políticos. Esta fase termina con la toma de una decisión. 
4. Fase programática: en este momento se lleva a cabo lo que se decidió en la fase política.

5. Fase de valoración: en la fase final se evalúa si la política pública que se ha llevado a cabo satisface las expectativas que los grupos habían depositado en ella. Dicho procedimiento puede conducir a una reconsideración del problema $y$, por tanto, a volver a comenzar el ciclo de formación de opinión partiendo de la experiencia anterior.

En puridad es en las tres primeras fases (de problema, de propuesta y política) en las que asistimos a un mayor proceso de conformación de la opinión pública, aunque si por algo se caracteriza precisamente la opinión pública es por su continua evolución y redefinición a lo largo de todas las fases. Otro hecho a tener en cuenta es que no resulta sencillo compartimentar la realidad social en fases que al acabar den paso a una siguiente etapa. El sistema político precisamente es tal por los procesos de retroalimentación a que da lugar cada una de las respuestas del sistema, que a su vez cambian las percepciones de los actores y ajustan sus estrategias nuevamente. De igual manera, como parte del sistema en la generación y canalización de demandas, la opinión pública es un continuum, un hecho social con idas y venidas que no se ajustan a rígidos cortes en el tiempo. Asumiendo estas precisiones, las fases que proponen estos autores nos resultan válidas para poder comprender el establecimiento de la agenda ciudadana.

Este ciclo de cinco fases también se ha visto en otros autores. Así, Downs (1972) identifica para el tema de la ecología un ciclo de atención (issue-attention cycle) sobre las cuestiones públicas en el que en un primera fase de preproblema se perfila el problema desde una perspectiva personal; una segunda de alarma por el descubrimiento de éste y de entusiasmo por las posibilidades de solución; una tercera en la que se percibe el coste real de hacer progresos significativos; una cuarta en la que el interés del público decae; y finalmente una quinta en la que el problema queda aparcado pero listo para poder relanzarse bajo una nueva forma.

\subsection{Estudios sobre la percepción de las agendas a nivel infraestatal}

Mientras que el proceso de conformación de las agendas ha recibido bastante atención por parte de los académicos, no lo ha recibido tanto su grado de interacción cuando consideramos dichas agendas en un entorno multinivel.

Spierkel (1981) investiga a través de un análisis del contenido la cobertura mediática del referéndum de 1980 por parte de la prensa regional de Québec. Esta autora muestra que, cuando hay un sentimiento regional intenso, marcado en este caso por el cleavage lingüístico, se produce una fuerte disociación de las agendas nacionales 
y regionales (Spierkel, 1981: 74). Por su parte, Doroshchuk y Mantashashvili (2016) estudian para la República de Tatarstan, parte de la Federación Rusa, el impacto que tiene la agenda mediática federal en la agenda mediática regional. En sus conclusiones observan que "el canal de televisión federal se caracteriza por el predominio de la agenda pública, mientras que las agendas personales y grupales manifestadas al cubrir temas locales y la proyección parcial de los problemas de todo el país en la región son específicos del canal de televisión regional" (Doroshchuk y Mantashashvili (2016: 62).

Ya dentro de estudios de agendas regionales hechos para España, Canel et al. (1996) analiza el primer nivel de la agenda setting en el ámbito local de la ciudad de Pamplona. Muestran que hay una correlación entre las noticias reportadas en los medios y la configuración de la agenda medida a través de encuesta. En este caso había un correlato entre la agenda local y las noticias locales, con menor prevalencia de cuestiones de ámbito nacional. Además, siguiendo a Zucker (1978), señalan la importancia de dos tipos de temas distintos que califican como obtrusive y unobtrutive. Los temas obtrusive son aquéllos que el ciudadano puede experimentar directamente, mientras que los segundos, para poder hacerlo, debe ser a través de los medios de comunicación. Detectan que el mayor poder de los medios se produce en los temas de tipo unobtrusive, frente a los primeros, pues los medios son la única fuente de información de que disponen.

Hay otros estudios que tratan estas cuestiones para el caso de Castilla y León. Mientras que Cheng et al. (2008) muestran el modo en que se presenta la información relacionada con la inmigración, Gómez-Limón et al. (2007) atienden a la percepción pública del problema de la despoblación del medio rural. Martínez-Sanz et al. (2015) destacan el segundo orden que tiene Castilla y León en las propias noticias que se dan sobre la región en periódicos de ámbito nacional vendidos en dicha región. Destacan que la aproximación informativa es más de corte provincial que regional propiamente dicha (Martínez-Sanz et al., 2015: 16). En esta línea, Hernández (2010: 16) subraya que Castilla y León es una comunidad autónoma que tiene una baja conciencia regional. Sus niveles de conciencia regional fueron bajos desde que comenzó su andadura como comunidad autónoma y se han mantenido así a lo largo de todo el período. Tal y como señala el estudio de Hernández (2010: 39), el bajo desarrollo de una conciencia regional en Castilla y León se debe a que ésta comunidad autónoma no ha conocido un desarrollo económico desde que se constituye como comunidad autónoma equiparable a otras comunidades, haciendo que su conciencia regional sea baja y en continua disputa. 


\section{Marco metodológico}

El estudio de la agenda-setting se ha centrado en los medios de comunicación y su relación para fijar la agenda ciudadana, pero menos atención se ha prestado a ver cómo los propios ciudadanos articulan dichas agendas cuando responden a las preguntas de las encuestas. Por ello, en este estudio presentamos primero nuestra pregunta de investigación sobre este campo menos estudiado que es el de la interacción de las agendas en función de los niveles de gobierno. Luego presentamos las técnicas que hemos empleado y, por último, nuestro marco espacial y temporal.

\subsection{Pregunta de investigación e hipótesis}

Para plantear nuestro estudio partimos de tres preguntas de investigación de las que se derivan todo nuestro diseño metodológico: qué elementos son los que conforman la agenda ciudadana de Castilla y León, cuáles de ellos han sido integrados en la agenda pública y en qué medida el cumplimiento de dicha agenda ciudadana tiene reflejo en la percepción sobre el desempeño de las instituciones políticas.

Para responder a estos interrogantes, partimos de la siguiente respuesta hipotética. Las condiciones materiales de vida influyen en la percepción de las situaciones como problemáticas y ésta en las opiniones que los ciudadanos expresan. Desde el momento en que los ciudadanos experimentan una serie de condiciones de vida $y$ perciben una situación como problemática, en primer lugar, aparece un grupo de ciudadanos no coordinados entre sí que perciben personalmente la situación como problema que requiere una atención pública. Estos ciudadanos funcionan como masa crítica sobre la que, con el paso del tiempo y ante la ausencia de solución de una situación vivida y percibida como problemática, se asienta la idea de que el problema tiene una dimensión pública para poder tener solución. En este momento el problema comienza a cobrar una dimensión pública, lo cual comporta la aparición de fórmulas de presión para que el problema entre en la agenda política.

Cuando un problema entra dentro del circuito público de comunicación, los medios funcionan como una "caja de resonancia": amplían el número de posibles destinatarios del mensaje, llegando a públicos cuyas condiciones de vida no hacen que se planteen el asunto en cuestión como un problema personal. Se produce en este caso un efecto llamado "de contagio" (Gil Calvo, 2003: 212-214), que provoca que personas que no viven un determinado asunto como un problema consideren que este tema (issue) deba ser parte de la agenda pública. De esta manera, en resumen, la cuestión comienza siendo percibida como un problema personal por un grupo de individuos que sufren la situación concreta. Posteriormente, la percepción de este 
problema se extiende y comienza a cobrar una dimensión pública, "contagiando" a grupos de población que no perciben el asunto como un problema personal.

Si bien éste es el funcionamiento esperado habitual, observamos que el análisis de la materia requiere tener en cuenta cuestiones adicionales. Así, y en especial en el caso de Castilla y León dada la ausencia de sentimiento regional, los ciudadanos, ante tal situación de desapego, desarrollan una serie de percepciones referenciales que no son propias, es decir, se asume un discurso que no se corresponde con sus condiciones materiales de vida. Por ponerlo en términos de agendas, los ciudadanos no responden a una agenda ciudadana propia sino a una agenda de referencia. En el caso de Castilla y León esto se produce con la agenda nacional. A esto se une el refuerzo mediático que hace que en los medios de comunicación primen dichas cuestiones, pero estos aspectos los dejamos fuera de este análisis, si bien reconocemos su importancia tal y como muestran otras investigaciones en la materia.

\subsection{Método y técnicas de análisis empleadas}

Para abordar esta investigación hemos optado por una triangulación metodológica, adaptando la metodología adecuada para cada elemento de la pregunta de investigación a partir de una evaluación de objetivos a alcanzar y recursos disponibles. El método estadístico y el comparativo son los que cumplen mejor estas tareas (Soto, 2015: 72). Para este estudio adoptaremos la variante del método comparado en su faceta de estudio de caso (Liijphart, 1971: 691). Ambos métodos muestran una gran compatibilidad, distinguiéndose fundamentalmente en la cantidad de casos analizados, y serán los que empleemos, valiéndonos del estudio de caso de tipo cualitativo para poder confirmar o refutar nuestra hipótesis.

Las técnicas empleadas son, principalmente, la aplicación de técnicas cuantitativas y cualitativas de análisis. Para realizar este estudio nos hemos servido de estudios preelectorales y postelectorales del Centro de Investigaciones Sociológicas, de las elecciones autonómicas de 2015, el barómetro SOCYL y encuestas preelectorales de las elecciones autonómicas de 2019, efectuadas por SYM Consulting y Sigmados. En concreto los estudios del CIS que hemos manejado son dos. El primero de ellos, el estudio 3.072, se trata de un estudio preelectoral con una muestra amplia representativa por cada una de las provincias de la comunidad autónoma. Son 3.000 encuestas presenciales recogidas en 191 municipios de las nueve provincias con un margen de error para el supuesto de un muestreo aleatorio simple de $1,8 \%$ a un nivel de confianza del 95,5\%. El segundo de los estudios, el número 3.094, ha sido diseñado para 1.000 entrevistados en persona, repartidos por todo el territorio. En el supuesto de un muestreo aleatorio simple, para un nivel de confianza del 95,5\%, el margen de error es del $3,2 \%$. 
Las técnicas de análisis que hemos empleado son de dos tipos. Por un lado, hemos empleado técnicas de estadística descriptiva, es decir, hemos expuesto la relación de los resultados a partir de la recogida de datos, el cruce entre ellos y la descripción de los patrones observados. Por otro lado, hemos llevado a cabo una inspección cualitativa de los datos. Por otro lado, hemos empleado la técnica del grupo de discusión. Consiste en la reunión de un grupo especialmente diseñado de 6 a 10 personas desconocidas entre sí que han discutido en torno a los temas que les hemos presentado. Hemos procedido a grabar las discusiones que han mantenido sobre los temas más relevantes de cara a las elecciones de mayo de 2019. Hemos ido dejando que el grupo aflore la corriente discursiva para poder ver las tendencias sociales que reflejan los perfiles elegidos. En el siguiente apartado detallaremos cómo se ha seleccionado la muestra y qué resultados se han obtenido en dicha selección.

\subsection{Marco temporal y espacial y selección de la muestra}

El objeto de estudio es el conjunto de la Comunidad de Castilla y León. Dada la imposibilidad de abarcar un estudio en cada una de las provincias que integran la comunidad castellanoleonesa, se ha optado por reducir el trabajo de campo a tres grupos de discusión. La selección de las provincias corresponde a tres criterios:

- Las tendencias de identificación regional.

- La representatividad sobre el conjunto de la Comunidad Autónoma.

- La provincia donde el voto se encuentra más fragmentado.

Otra cuestión es el marco temporal. Temporalmente circunscribimos el estudio al trabajo de campo que hemos realizado entre septiembre y diciembre de 2018. Únicamente se toma como referencia las elecciones autonómicas de 2015 que es la cita electoral previa a las nuevas elecciones a Cortes de Castilla y León celebradas en mayo de 2019.

Para la configuración de nuestros grupos de discusión se seleccionaron a participantes mayores de 18 años que residen en Castilla y León. Concretamente se han confeccionado tres grupos de discusión. El primero de ellos estuvo compuesto por estudiantes residentes en Castilla y León (GD1) pero que cursan sus estudios fuera de la comunidad autónoma, el segundo por ciudadanos de la provincia de Burgos (GD2) y, el tercero, por habitantes de la provincia de León (GD3). La selección se realizó teniendo en cuenta varios criterios. El primer grupo de discusión buscaba tener una representación de personas de la mayor parte de provincias, especialmente de Segovia y Ávila, aunque no residentes en ese momento, que provenían de entornos rurales. El segundo grupo tenía por objetivo mostrar las opiniones de un entorno 
urbano. El tercer grupo se dirigió a la provincia de León pues presenta una peculiaridad con respecto al resto de provincias y es su marcada identidad.

El método utilizado para contactar con los potenciales participantes es una "variedad de muestreo no probabilístico" (Cea D'Ancona, 1996: 202) denominado "bola de nieve" del que se van escogiendo a los participantes "a partir de las referencias aportadas por los sujetos a los que ya se ha accedido" (Cea D'Ancona, 1996: 202), por lo que este proceso realizado repetidamente hace que la muestra vaya aumentando como una "bola de nieve". Este tipo de método es muy útil cuando en la "población por sus propias características son difíciles de identificar y de localizar" (Cea D'Ancona, 1996: 202). En este caso los participantes potenciales sirven para incorporar nuevos elementos al estudio y "la localización de uno de los integrantes de la población se vuelve doblemente valiosa al suponer la identificación de otras personas pertenecientes a la misma población" (Cea D'Ancona, 1996: 202). Para ello se pregunta a los sujetos ya "descubiertos" si conocen a otros en su misma situación (Cea D'Ancona, 1996: 202).

\section{Principales problemas para los ciudadanos en Castilla y León}

En este apartado se realizará un análisis de los datos facilitados por las encuestas postelectoral del CIS del 2015 (estudio no 3094), la encuesta del CIS (estudio no 3231), la encuesta preelectoral del CIS de 2019 (estudio n 3245) y el barómetro de la Asociación Profesional de Sociología de Castilla y León de primavera de 2016, por ser los últimos estudios efectuados en los que se pueden identificar los problemas que afectan a los ciudadanos de Castilla y León antes de la cita electoral autonómica de 2019. Estos resultados serán complementados y contrastados con la información obtenida a través del trabajo de campo efectuado a través de los grupos de discusión. De esta manera, se podrá determinar cuáles son los principales problemas de los ciudadanos de esta comunidad autónoma, y cuál es su percepción sobre los mismos.

\subsection{Principales problemas según las encuestas del CIS}

Según los datos del CIS en su encuesta postelectoral del 2015 (Estudio n 3094), los ciudadanos de Castilla y León consideraban que los problemas más importantes de su comunidad eran, por orden de importancia, la creación de empleo, la sanidad y la educación, que se encontraban muy destacados del resto, como se puede comprobar en la tabla 1. 
Tabla 1.

Temas más relevantes para el electorado castellanoleonés en 2015

\begin{tabular}{lccc}
\hline \hline & Primer lugar & Segundo lugar & Total \\
\hline La educación & 17,9 & 23,6 & 41,5 \\
\hline La sanidad & 14,8 & 36,5 & 51,3 \\
\hline La vivienda & 1,7 & 6,4 & 8,1 \\
\hline La creación de empleo & 60,8 & 16,2 & 77,0 \\
\hline La seguridad ciudadana & 0,2 & 1,0 & 1,2 \\
\hline La inmigración & 0,1 & 1,7 & 1,8 \\
\hline La protección del medio ambiente & 0,2 & 1,0 & 1,2 \\
\hline Las infraestructuras (carreteras, ferrocarriles) & 0,3 & 2,6 & 2,9 \\
\hline Otros & 1,0 & 3,9 & 4,9 \\
\hline N.S. / N.C. & 2,9 & 7,0 & 9,9 \\
\hline (N) & $(990)$ & $(990)$ & \\
\hline \hline
\end{tabular}

Fuente: Estudio CIS no 3094.

En este mismo sentido, hay que mencionar, que más de tres cuartas partes de las personas consultadas destacan como el mayor problema de esta comunidad autónoma la creación de empleo, más de la mitad de las personas entrevistadas consideran que es la sanidad, mientras algo más del $40 \%$ piensan que es la educación. Otras cuestiones como la vivienda, la seguridad ciudadana, la inmigración, la protección del medio ambiente o las infraestructuras apenas presentan cifras significativas.

Si a su vez tomamos los datos del barómetro efectuado por la Asociación Profesional de Castilla y León (SOCYL, 2016) en la primavera de 2016, se puede comprobar que los problemas de los ciudadanos de esta comunidad, son prácticamente los mismos que los señalados por la encuesta del CIS. El modelo económico y productivo es la primera cuestión que preocupa a los castellanoleoneses, la segunda es el desempleo, la tercera la situación demográfica, la cuarta la desafección política y la corrupción, y por último, los servicios sociales y sanitarios. 
Tabla 2.

Problemas de los ciudadanos de Castilla y León

\begin{tabular}{|l|c|}
\hline & Problemas \\
\hline Desempleo & $94,6 \%$ \\
\hline Modelo económico y productivo & $73,0 \%$ \\
\hline Situación demográfica & $41,8 \%$ \\
\hline Desafección política y corrupción & $26,0 \%$ \\
\hline Servicios sociales y sanitarios & $17,3 \%$ \\
\hline
\end{tabular}

Fuente: Barómetro de SOCYL.

Ahora bien, también es necesario trasladar este estudio hacia la percepción que tienen los castellanoleoneses sobre los tres principales problemas que afectan a España durante el mismo periodo de tiempo. Comparando la percepción de los residentes en Castilla y León con el resto de los españoles sobre los problemas que afectan a España se podrá comprobar si la respuesta anterior es intrínseca a la propia comunidad, o si es reflejo de la situación por la que ha atravesado el país.

Tabla 3

Problemas de España para los residentes de Castilla y León en comparación con el resto de españoles

\begin{tabular}{|c|c|c|c|c|}
\hline & \multicolumn{2}{|c|}{ Noviembre de 2018} & \multicolumn{2}{|c|}{ Abril de 2019} \\
\hline & $\begin{array}{l}\text { \% Castilla y } \\
\text { León }\end{array}$ & $\begin{array}{c}\text { \% total para } \\
\text { España }\end{array}$ & $\begin{array}{c}\text { \% Castilla y } \\
\text { León }\end{array}$ & $\begin{array}{c}\% \text { total para } \\
\text { España }\end{array}$ \\
\hline El paro & $58,5 \%$ & $58,5 \%$ & $64,1 \%$ & $61,5 \%$ \\
\hline La corrupción y el fraude & $31,0 \%$ & $29,4 \%$ & $33,0 \%$ & $31,5 \%$ \\
\hline $\begin{array}{l}\text { Los/as políticos/as en general, los } \\
\text { partidos y la política }\end{array}$ & $26,9 \%$ & $31,3 \%$ & $31,9 \%$ & $31,4 \%$ \\
\hline Los problemas de índole económica & $22,8 \%$ & $22,4 \%$ & $21,8 \%$ & $28,3 \%$ \\
\hline Independencia de Cataluña & $17,0 \%$ & $9,7 \%$ & $17,5 \%$ & $11,7 \%$ \\
\hline La sanidad & $8,2 \%$ & $10,8 \%$ & $18,8 \%$ & $17,0 \%$ \\
\hline La inmigración & $7,6 \%$ & $8,9 \%$ & $8,5 \%$ & $9,4 \%$ \\
\hline $\begin{array}{l}\text { Los problemas relacionados con la } \\
\text { calidad del empleo }\end{array}$ & $6,4 \%$ & $9,4 \%$ & $13,7 \%$ & $13 \%$ \\
\hline
\end{tabular}




\begin{tabular}{lcccc}
\hline \hline & Noviembre de 2018 & \multicolumn{2}{c}{ Abril de 2019 } \\
\hline \% Castilla y & $\begin{array}{c}\text { \% total para } \\
\text { España }\end{array}$ & $\begin{array}{c}\text { \% Castilla y } \\
\text { León }\end{array}$ & $\begin{array}{c}\text { \% total para } \\
\text { España }\end{array}$ \\
\hline Las pensiones & $5,8 \%$ & $6,9 \%$ & $9,8 \%$ & $10,7 \%$ \\
\hline Los problemas de índole social & $5,8 \%$ & $9,2 \%$ & $8,2 \%$ & $10,9 \%$ \\
\hline \hline
\end{tabular}

Fuente: Estudio $\mathrm{CIS}$ no 3.231 y estudio CIS no 3.245 .

Como se puede comprobar en la tabla 3, el paro continúa siendo la principal preocupación de los castellanoleoneses. La segunda cuestión en importancia es la corrupción y el fraude, e íntimamente conectado con lo anterior, los políticos, los partidos y la política en general. Mientras que la sanidad o la educación quedan bastante relegadas de las prioridades que debe afrontar el país, el motivo debe responder a que se tratan de preguntas espontaneas. Además, en la agenda de los castellanoleoneses están presentes cuestiones de ámbito nacional como la independencia de Cataluña.

Cuando comparamos los datos autonómicos con los nacionales se destaca que Castilla y León tiene una fuerte agenda nacional y una agenda autonómica mucho más limitada. En este sentido, en las encuestas del CIS no aparecen temas como la despoblación cuando se pregunta por los problemas de la región, pero sí afloran en el Barómetro de SOCYL. Asimismo, las preocupaciones principales se centran en cuestiones como el paro, la corrupción o la cuestión catalana que una agenda de la región propiamente dicha, como podría ser la despoblación. Además, temas como la educación, la sanidad, las pensiones o la calidad del empleo tienen una menor importancia y los ciudadanos castellanoleoneses no parecen trasladar sus agendas ciudadanas a las agendas nacionales.

De esta manera, la agenda ciudadana se puede componer de numerosos temas, aunque solo unos pocos pueden ser considerados como relevantes. De hecho, cinco son los que han tenido reflejo en todas las encuestas: el empleo, la situación económica, la sanidad, la corrupción política y la educación. Son en definitiva problemas que forman parte de la agenda casi de manera permanente. Otros son temas que forman parte de la agenda de manera intermitente, es decir, es lo que Downs (1972: 41), señala "cuestiones que surgen de repente sobresalen, llama la atención durante un breve periodo de tiempo y luego -muchas veces sin encontrar solución- desaparece paulatinamente", como es el problema de la despoblación, las infraestructuras y la 
comunicación, siendo éstos precisamente los que afectan de manera más particularizada a los ciudadanos de Castilla y León.

\subsection{Percepción ciudadana sobre los problemas de su comunidad autónoma}

Los problemas que se detectaron mediante los grupos de discusión con ciudadanos castellanoleonés fueron similares a los de las encuestas del CIS. En este sentido, hay que mencionar que la situación económica y la creación de empleo, tal y como se comentaba en el apartado anterior, continuaban siendo las dos cuestiones que más les preocupan, aunque también se ha podido detectar un cierto descontento con la gestión del gobierno de la Junta de Castilla y León durante estos últimos años, y que encuentra su plasmación en problemas como el desarrollo económico desigual, la despoblación y el envejecimiento de la población, y la falta de infraestructuras. Todos estos temas afloraban a lo largo del discurso y no de manera espontánea, si no que aparecían a medida que avanzábamos en el propio grupo de discusión. Gracias al análisis de los discursos producidos se puede exponer de una manera más profunda los problemas que afectan a la agenda ciudadana en esta comunidad autónoma. Después se podrá comprobar si los elementos que integran esta agenda han sido recogidos a juicio de los ciudadanos castellanoleoneses.

\subsubsection{Desarrollo económico desigual}

El fomento del empleo es uno de los dos retos más importantes a los que tiene que hacer frente en Castilla y León para reanimar la comunidad autónoma. Si se toman como referencia los datos de la Encuesta de Población Activa (EPA) del primer trimestre de 2019 la tasa de desempleo en esta comunidad es del 12,42\%, dos puntos por debajo de la tasa en España. Buena parte de este empleo se halla en la agricultura y la ganadería, lo que genera, como se podrá ver más adelante, otros problemas como son la fuga de jóvenes, el desempleo, la precariedad laboral y la emigración. Esto se produce, principalmente, porque el colectivo de personas con especialización en servicios registra una tasa de paro del $21,4 \%$, es decir, una de cada cuatro personas con formación en el sector terciario en la comunidad está en desempleo. A ello hay que añadirle que las Tecnologías de la Información y la Comunicación (TIC) sitúan su tasa de desempleo en el 13,1\%, según los datos de la EPA. Esto significa que, según el Instituto Nacional de Estadística (INE), casi la mitad de los ocupados en esta comunidad tiene una formación básica, hecho que no guarda relación con el nivel de estudio que presenta la población de la misma para el conjunto del país. Tal es así, que, en virtud de los datos de la EPA, la tasa de desempleo en TIC en Castilla y León es superior a la media de España, un 13,1\% frente al 9,2\%. 
En cuanto al desarrollo económico hay que señalar el Producto Nacional Bruto (PIB) es de 58.817M, lo que le sitúa como la séptima economía de España por volumen del PIB, mientras que cuanto al PIB per cápita, que es un buen indicador de la calidad de vida, en Castilla y León en 2018 es de 24.397€, lo que le pone en el octavo lugar del ranking del PIB per cápita en España.

Este es el panorama de la situación económica y desempleo por la que atraviesa la comunidad, y estos datos han sido corroborados por la percepción que tienen los ciudadanos que han participado en los grupos de discusión. Para ellos, la economía de la Castilla y León rural está basada en el sector ganadero y agrícola y sostenida, en buena parte, por los fondos europeos. La economía de las cuencas mineras se ha venido desarticulando y los fondos europeos (MINER) para la reestructuración del sector no parece que hayan sido utilizados con éxito. La industria ha ido desapareciendo de muchas zonas y los planes de industrialización no parece que sean muy viables por la falta de atractivo de la comunidad. Varios son los factores mencionados como inhibidores del emprendimiento:

- Pérdida de población, que supone menos "potenciales clientes" para los productos o servicios.

- Trabas desde la Administración Pública, con un exceso de burocratización para empresarios.

- Ausencia de políticas realmente favorecedoras del emprendimiento.

- Carencia de una red de comunicación interregional en la comunidad que desincentiva la creación de iniciativas empresariales.

- Peajes, que lastran con costes adicionales a las empresas.

- Falta de infraestructuras modernas que integren todas las partes del territorio.

A esto hay que sumarle que en las zonas urbanas se ha producido una fuerte terciarización del empleo y la precarización del mismo, sobre todo en el sector de la hostelería y la restauración. Las condiciones de trabajo son cada vez más coercitivas, lo que lleva a afirmar que "la gente que no emigra se queda a trabajar de camarero" (GD3).

\subsubsection{Despoblación, envejecimiento y fuga de jóvenes de la comunidad}

Se trata de uno de los temas más recurrentes en el discurso de los grupos. La despoblación se vive como un drama en las zonas rurales y en las zonas urbanas el tema de la pérdida de población y el éxodo joven es una preocupación real para los padres y las madres. Desde 2011 el saldo migratorio muestra una perdida cercana a los 60.000 habitantes, según datos del INE. Pero lo grave es que 3 de cada 4 habitantes tenía una edad de entre 25 y 44 años y la mitad entre 5 y 34 años. Esto pone de relieve 
a su vez otro problema, el envejecimiento de la población, convirtiendo a Castilla y León en una de las comunidades con más población mayor.

En los pueblos se produce una movilidad de gente joven que, inicialmente, sale a estudiar a otras capitales de provincia dentro de la comunidad o se desplaza a otras comunidades para ello y que, después, difícilmente regresan a sus pueblos porque allí no existe ninguna oportunidad laboral acorde con una mayor cualificación. Las ciudades tampoco ofrecen muchas expectativas laborales a esta población joven, formada y cualificada, por lo cual la juventud de esta comunidad autónoma busca otras alternativas en otras comunidades o en el extranjero. La poca variedad formativa en estudios superiores también contribuye a la anticipación de esta fuga de jóvenes.

“- Todos tenemos hijos y lo vemos. Porque vemos que en un momento determinado se van a ir.

- Es que te tienes que ir.

- Primero por estudiar, la universidad aquí, en Valladolid, en Salamanca, más o menos las opciones están cubiertas, pero después es que laboralmente posiblemente tengan que salir, y a parte que ves que es bueno para su educación pero lo malo es que no vuelven. Es que si salen ya no vuelven.” (GD2)

La falta de políticas que fijen a la población joven en el territorio está ocasionando un éxodo joven. Hoy por hoy, Castilla y León no es un lugar para jóvenes. La aspiración de la juventud de las zonas rurales es salir. Los pueblos no tienen una oferta cultural ni laboral que atraiga para echar raíces;

“Todos los jóvenes que yo he conocido, cuando estaban en el instituto, en mi pueblo o alrededores, lo que principalmente querían era marcharse porque no se ofrece gran cosa, ni a nivel cultural, ni a nivel laboral... y lo laboral que se ofrece es sobre todo agrario, agrícola y creo que los jóvenes lo que buscan es un poco más de formación influidos por sus padres y demás..." (GD1)

Por otro lado, muchas zonas urbanas donde no hay oferta universitaria están sufriendo el mismo proceso, debido a la falta de atractivo cultural y social para la población joven. Esto lleva a que la población más dinámica sea la que tienda a marcharse de la comunidad, perdiendo ese capital humano y cultural con dicha marcha.

"Todo el mundo está deseando acabar bachillerato para irse de la ciudad en la que estamos, por eso, por lo cultural, porque no hay nada nuevo que nos motive a quedarnos y todo el mundo quiere irse a Salamanca, Madrid y otros sitios" (GD1).

Existe un proceso de estigmatización de la población joven que permanece en la localidad de origen. Se destaca un sentimiento de inferioridad de la escasa población 
juvenil que se ha quedado y no ha estudiado fuera de su localidad. La frustración del desempleo en este colectivo es mayor.

“-...de hecho si te quedas, yo lo he comentado muchas veces, si te quedas eres un...

-Un pringao.

- Eso, un pringao, o si te quedas porque tienes unos vínculos familiares o tus padres no pueden pagarte o lo que sea y si te quedas por gusto de quedarte eres raro." (GD1)

Se asume de manera natural la movilidad de la juventud por estudios y formación, pero la falta de políticas de retorno efectivas para que los jóvenes formados tengan oportunidad de regresar y motivos para hacerlo es un factor criticado. Hay un alto grado de pesimismo sobre el futuro de la juventud en la comunidad, aunque, desde las propias administraciones públicas, se favorece las salidas profesionales al exterior pese a que eso perjudica la estabilidad de la población. Así, se proponen acciones para cubrir necesidades formativas que faciliten la salida de la juventud a terceros países, cursos de idiomas por ejemplo, y, sin embargo, no hay políticas que fijen población joven al territorio para asegurar el relevo generacional.

"Yo veo mi vida aquí pero no veo la de mis hijos. Me da mucho miedo, me da mucho miedo mis hijos. Yo les estoy inculcando para que hablen dos idiomas porque yo sé que aquí no se van a quedar. (...) Yo que trabajo con jóvenes los más brillantes de cada promoción se van y es que si se quedan aquí me dan pena. Ése es el problema." (GD3)

El retorno joven es incierto. Las personas jóvenes que han salido para cursar estudios y se han especializado no encuentran oportunidades laborales en la comunidad, sobre todo hablando de trabajo asalariado. Se percibe que las políticas territoriales que se vienen haciendo por parte de la Junta de Castilla y León se centran en la agricultura y la ganadería, un sector poco atractivo para la gente joven, y los planes de industrialización no están dando mucho fruto.

Una vez que la gente joven ha salido a estudiar fuera no suele regresar porque tiene una cualificación superior a la que el mercado laboral de la comunidad ofrece. La falta de expectativas es menor para los jóvenes más formados. No hay oportunidades para personas cualificadas. El regreso de las personas jóvenes con estudios superiores al ámbito rural supondría una renuncia a la carrera profesional y una vuelta a la actividad ganadera o agrícola familiar “...pero al final tienes que dedicarte a una cosa que no has estudiado, ni quieres” (GD1). Por ello, las personas jóvenes que han salido para estudiar no ven oportunidades para el regreso, sobre todo en poblaciones rurales y en pequeñas ciudades en proceso de pérdida de población y recursos. 
Retornar a la comunidad puede ser más fácil en las grandes capitales de provincia donde hay oportunidades dentro de las instituciones, por ejemplo.

"Volver al mismo pueblo es complicado. En 10 años ha pasado de 800 habitantes a tener 700 y se nota también en la calle, anda pues este año ya no han hecho no sé qué, o este año han dejado de hacer no sé cuál y... eso no va a tener nada que ver dentro de cinco años". (GD1)

Además de las escasas posibilidades laborales, otro freno importante para el retorno de la población joven emigrada son las escasas posibilidades culturales y sociales que las zonas rurales ofrecen y la pérdida paulatina de servicios y recursos de las mismas.

"En mi pueblo el Secretario (del Ayuntamiento) que había (y ya se ha) jubilado llevaba varios pueblos de la zona y sacaron la plaza y aunque me dijeron a mi si quería presentarme. ¡No! O sea, es que en el pueblo es lo que dices, no es sólo por el trabajo, es también por lo social, no hay nada." (GD1)

\subsubsection{Infraestructuras y comunicaciones}

La carencia de infraestructuras se destaca como un problema estructural en toda la comunidad, aunque este problema no se ha visto reflejado en las anteriores encuestas. Aunque el progreso de las telecomunicaciones se ha valorado y está llegando la red a casi todo el territorio, el transporte y las carreteras siguen siendo deficitarias en la comunidad y un factor que retrasa e inhibe el desarrollo económico de determinados territorios. Se destacan tres problemas clave: servicio de transporte público deficitario, la comunicación por carretera marca la diferencia de desarrollo económico territorial y la falta de una red de comunicación interna que hace que muchas de las zonas estén desconectadas y aisladas.

Están desapareciendo cada vez más servicios de transporte público de los pueblos, dejando a las zonas rurales aún más incomunicadas y la red de tren no cubre las necesidades de conexión. También se considera importante tener una conexión de transporte público y carretas sin peaje con otras provincias. Por ejemplo, entre Ávila y Madrid están a una corta distancia, pero sin embargo, no hay tren, y solo una compañía de autobús hace este trayecto. Lo mismo sucede con otras comunidades limítrofes como Cantabria y País Vasco, donde hay líneas regulares directas.

"En el de línea tardas tres horas y en el coche tardas una hora. Mis amigos no vienen. Si estás estudiando en Santander no vas a venir a Burgos, ¿para qué? ¡Tardas tres vidas en llegar!’(GD1)

Esto marca una tendencia que muestra distinta configuración socioeconómica en función del acceso a grandes vías de comunicación. Las localidades cercanas a la 
autovía tienen un mayor desarrollo industrial, las más alejadas están abandonadas. La cercanía a la autovía marca el grado de desarrollo de las localidades, es donde está toda la industria de transformación agroalimentaria, la mayoría de los servicios, etc. Esto tiene un doble efecto de distinta configuración socioeconómica y, por tanto, distinta tendencia electoral y, al mismo tiempo, diferencia sus discursos a pesar de tener condiciones territoriales similares. En este sentido, las carreteras de peaje están siendo un lastre para el desarrollo de determinados territorios, que encuentran excesivo su pago, llegando a afirmar que "estamos cerrados por el peaje" (GD1).

Por otra parte, la falta de una red de infraestructuras que conecte a las diferentes localidades arrastra al aislamiento a las pequeñas localidades y dificulta las relaciones entre las distintas provincias, no favoreciendo el sentimiento identitario de comunidad. Se ha venido desarrollando un modelo de comunicación centralista representado arquetípicamente por el AVE, frente a un modelo interregional representado por FEVE. Las políticas de la comunidad han proyectado infraestructuras como el AVE que fomentan las conexiones centralistas desde Madrid e impulsan el turismo, pero que no conectan a la comunidad ni establecen relaciones reticulares dentro de la sociedad castellano-leonesa. Las malas comunicaciones en la periferia hacen que sea más fácil llegar a Madrid que a otra capital de provincia, desplazando la actividad hacia Madrid en detrimento de otras zonas urbanas de la comunidad.

"El FEVE se está dejando caer a pedazos. Aquí viene Vicente Herrera y se da en el pecho con la despoblación rural pero lo que tienes que hacer es articular. Por ejemplo, aprovechar unas infraestructuras que tienes creadas para que la gente pueda venir a León desde los pueblos. Se va a desmantelar el FEVE no tardando 1 ó 2 años. Ya no hay ni revisor para que no tengamos ni datos de las estadísticas de uso de usuarios." (GD3)

\subsubsection{Lucha contra la corrupción}

Se considera que especialmente Castilla y León tiene un problema muy serio de corrupción en las instituciones. La corrupción se define como el mal endémico de la comunidad, una enfermedad muy difícil de erradicar en la política. Se ha enraizado tanto, que se percibe como una acción institucionalizadada debido, sobre todo, a la falta de alternancia política del partido en el poder (PP). No obstante, la corrupción está normalizada por la ciudadanía bajo el argumento de que es una cualidad de los partidos políticos en el poder. La corrupción es un tema recurrente en los discursos de los perfiles de mediana edad en donde se expone una actitud muy visceral e indignada. No obstante, la gente joven no encuentra este problema como una argumentación política de peso en la oposición y buscan programas con políticas activas reales. 
La corrupción en Castilla y León se entiende, no como un conjunto de hechos aislados, sino como una entidad o círculo vicioso/círculo dominador: Un grupo de individuos que comparten intereses en un espacio común y están presentes en las esferas política, económica y mediática. Este círculo es difícil de romper porque tiene una fuerte presencia y poder en los sectores más determinantes:

“- Es una economía circular.

- Incluso solidaria.

- Muy solidaria con ellos y muy insolidaria con el resto de la población. (GD2)

"En Castilla y León hay una mafia brutal que todos conocen. Los caciques siguen existiendo en nuestra comunidad. En esta ciudad existen (Burgos). Tienen el control de medios de comunicación, que tienen su trama clientelar y que se abastecen, se autoabastecen." (GD2)

La ciudadanía define la corrupción como un lastre para el progreso y desarrollo económico de la comunidad, pero sobre todo para el desarrollo de políticas más sociales. Cabe señalar que la corrupción se percibe como la causa concreta de la pérdida de recursos económicos para el desarrollo de políticas sociales que atiendan a la ciudadanía. Es por eso que se tiene la sensación de que la sanidad tiene cada vez menos recursos y peores servicios, ya que uno de los casos más señalados de corrupción es el del Hospital de Burgos.

\subsection{Percepción ciudadana sobre políticas públicas específicas}

En el apartado anterior analizamos las cuestiones que percibían los participantes como estructurales, es decir, cuestiones que requieren cambios a largo plazo, grandes inversiones e incluso la modificación de conductas instaladas en la comunidad autónoma. En este apartado trataremos cuestiones más específicas como desempleo, precariedad laboral y pobreza laboral, sanidad, educación, vivienda, medioambiente e inmigración.

\subsubsection{Desempleo, precariedad laboral y pobreza laboral}

El desempleo es el problema más destacado en las ciudades. En el discurso emerge como un fantasma del que se huye. La gente joven se va de la comunidad para no sufrirlo y el problema de quien lo experimenta sufre es su cronificación dada la falta de alternativas con las escasas expectativas de generación de empleo en la Comunidad. Así, si bien Castilla y León mantiene una tasa de desempleo por debajo de la media nacional y los participantes de nuestros grupos no se encontraban en 
su mayoría afectados directamente, sí se percibía como problema por el fenómeno generado de la expulsión de población.

La precariedad laboral y la pérdida de derechos laborales es la otra cara del empleo en la comunidad. Se matiza, sobre todo entre las mujeres, la discriminación laboral por motivos de género que se vive en la comunidad y la fuerte precarización del empleo en cuanto a subocupación por insuficiencia de horas, temporalidad, sueldos bajos...

En el discurso del grupo de Burgos se vislumbra la presencia de la pobreza laboral como una circunstancia habitual entre las personas trabajadoras que sufren las consecuencias crónicas de la precarización en el empleo y deben acudir a las ayudas familiares para poder afrontar las necesidades más básicas. En este sentido se destaca la creación de una economía circular y solidaria de las familias que se atienden y se sostienen:

"Yo no tengo problemas porque afortunadamente trabajé toda mi vida y me ha quedado un sueldo bastante majo (...) pero sí me preocupa el resto de la familia porque yo sé que ellos lo están pasando mal. Entonces tú intentas ayudar y no alcanzas para todos. Y yo digo ¿por qué tengo que ayudar yo si ellos están trabajando también? Pues sencillamente porque no les cubre los gastos, entonces todos andamos escasos. Andamos escasos." (GD2).

“QQué haríamos tantas? Yo, sin mi madre, ni mis hijos ni yo podríamos subsistir. He dicho que gano 610 euros y pago 500 de alquiler. ¿Vale? Y tengo un niño de 13 años y una niña de 7 . Es verdad que tienen una pensión de alimentos de su padre, pero con eso pagamos precisamente el alquiler, el resto corre de $\mathrm{mi}$ parte." (GD2)

\subsubsection{Sanidad, educación, vivienda y medioambiente}

Las políticas de sanidad, educación y vivienda han sido cuestionadas por los participantes. Aunque los problemas que plantea cada una de las cuestiones es diferente. La falta de cobertura sanitaria y en educación se presentan como un problema estructural en las zonas rurales. Sin embargo, en las zonas urbanas se habla de recortes y bajada de la calidad de estos servicios y de la falta de políticas sociales de vivienda.

La falta de cobertura sanitaria en el medio rural (falta de pediatras, médicos de familia...) obliga a los usuarios a desplazarse a otras localidades para recibir la atención sanitaria. En el caso de enfermedades crónicas o de vejez la falta de servicios sanitarios propicia la emigración a las ciudades y acelera los procesos de despoblación. En el ámbito urbano la cuestión se traslada a los recortes, la menor calidad de los servicios sanitarios públicos, los retrasos de las citas, las huelgas de médicos, la pérdida de calidad de las instalaciones... Se considera que el caso de corrupción y 
mala gestión del Hospital de Burgos ha favorecido el deterioro de la sanidad. Así, se afirma que el hospital de Burgos "es el vicio de la sanidad de Castilla y León", "la ruina de la sanidad de Castilla y León” (GD2).

En cuanto a la educación, no se cuestiona la calidad, ya que se considera que en Castilla y León se forma muy bien a los jóvenes. El castellanoleonés asume las políticas de colegios agrupados en las zonas rurales como solución de compromiso. También asume la movilidad joven dentro de la comunidad por motivos de estudios. Sin embargo, el descontento, sobre todo de la gente joven, viene por una falta de coherencia entre el nivel formativo que se ofrece y la falta de expectativas laborales en la comunidad. "forma bien a los jóvenes y los deja escapar, es como una contradicción." GD1. Las críticas se derivan hacia una falta de conexión entre la universidad y la empresa en la comunidad.

La vivienda supone un problema en las ciudades, principalmente por el precio del alquiler medio en relación al nivel medio de ingresos. Los alquileres en las capitales de provincia son muy elevados, si se tiene en cuenta el sueldo medio de la gente con menos recursos. El gasto medio de alquiler está por encima del umbral de endeudamiento límite por vivienda para un sueldo medio de 1000 euros en algunos casos.

"Yo vivo en Valladolid y con menos de 500 euros difícil (encontrar un alquiler), puedes encontrar algo, pero... ¿qué es lo que te queda? Coge transporte, facturas, comida..." (GD2)

No obstante, aun cuando surge en casos puntuales el tema de la vivienda, en general no hay una percepción de que ése sea un problema prioritario, pese a que se resalta en las estadísticas por parte de $8,1 \%$ de los encuestados. En ocasiones nos encontramos a un problema que es percibido como tal más por los colectivos que ya tienen una vivienda por una cuestión referencial, que porque sea su situación personal (Antón et al., 2007).

El medio ambiente es una cuestión que, aunque no vertebra el discurso, aparece de forma puntual y es señalada por las personas más activistas. Preocupan los vertederos no controlados en la comunidad y la protección medioambiental del territorio. A nivel local se hacen costar varias cuestiones puntuales a resolver.

"León es una zona proveedora de recursos naturales (energía, agua...) y a su vez recibe los detritos de todas las demás. Hay centros de tratamientos de residuos en León de neumáticos. El cáncer se queda aquí." (GD3) 


\subsubsection{Inmigración}

El discurso de la inmigración es residual: la inmigración no parece suponer un problema actual en la comunidad. Resulta ser más un discurso político del momento con fines electoralistas. Un tema traído del debate nacional pero en el fondo un problema en la comunidad que, aun así, tiene cabida en el discurso de los ciudadanos castellanoleoneses. Así, a pesar de tener un 5\% de inmigrantes en la región, por debajo de la media nacional y tres veces menos que la cifra más alta del 15\% de Baleares, es éste otro tema nacional que tiene cabida en la agenda ciudadana.

Cuando profundizamos en el grupo de discusión sobre el tema, el discurso se centra en la emigración y en las políticas que no se ocupan de fijar la población de Castilla y León, la emigración de gente joven al extranjero y a otras comunidades. El proceso migratorio se viene observando desde varias generaciones: "es lamentable, pero ves en generaciones de gente de 30 años que dicen -no, es que mi cuadrilla están todos por ahí (fuera de la localidad)." (GD2)

\section{Incidencia de la agenda nacional sobre la agenda ciudadana en Castilla y León}

La teoría relaciona la agenda pública con la agenda de los ciudadanos y de los medios de comunicación y convierte a éstos últimos en un verdadero determinante de aquélla, al menos en sus formulaciones iniciales (Tamayo y Carillo, 2005: 670). Pero no hay tantos estudios sobre la incidencia de las agendas en múltiples niveles de gobierno. Así, en los determinantes de la agenda ciudadana no se ha estudiado en qué medida las agendas ciudadanas al nivel regional son un reflejo de dichas agendas. En este estudio nosotros introducimos una cuestión adicional al considerar la relación entre agendas, que son los efectos cruzados entre los niveles.

Tal y como hemos visto en el apartado anterior, nuestros análisis a través de grupos de discusión ponen de manifiesto que en la región de Castilla y León resulta complicado acceder a las agendas ciudadanas, y que esto se produce tras un proceso de reflexión propio de la técnica de investigación que hemos empleado. Cuando hemos procedido a realizar las preguntas iniciales de manera genérica, el discurso dominante que han mostrado los ciudadanos castellanoleoneses ha girado hacia las cuestiones de ámbito nacional, incluso aunque las preguntas fueran de ámbito autonómico o personal. Esto muestra que, en determinados ámbitos regionales con una baja conciencia regional (Hernández, 2010), los discursos que recogen las encuestas reflejan en mayor medida dichas cuestiones, haciendo que el entrevistado asuma dichos discursos.

Observamos que el análisis de los grupos de discusión arroja otra cuestión reseñable y particular de Castilla y León, que es cierto carácter fatalista que lleva a la inacción. La percepción de los problemas se ve como algo irresoluble, de manera que 
esto hace que no haya una exigencia directa a los poderes públicos para su solución. En este sentido, los ciudadanos castellanoleoneses "despolitizan" algunas cuestiones no por dejar de encontrarlas relevantes, sino porque no ven que la política pueda darle una alternativa al problema. Así, la valoración de las instituciones es tan baja que esa inevitabilidad lleva a que no se exija y, por tanto, asuman en mayor medida como propias cuestiones de agenda que no lo son. En esta línea, resulta llamativamente interesante que la cuestión catalana sea mencionada en Castilla y León como uno de los problemas principales de España prácticamente el doble que en el resto de España y al mismo nivel que la propia Cataluña. Al comienzo de los grupos de discusión estos temas afloraron también y solo fue tras un trabajo de reconducción de los mismos hacia las cuestiones regionales que el tema se centró en Castilla y León. Así se refleja en nuestros grupos de discusión.

"Los medios de comunicación han hecho un buen trabajo y la gente está más preocupada de lo que pase en Cataluña o el Brexit que lo que pasa en Castilla y León. La gente conoce más a los consellers ésos de Cataluña que a cualquier consejero de Castilla y León. Al final es un caldo de cultivo que han sembrado fabulosamente bien y eso lo que va a hacer es que la gente vote al PP." (GD2)

Otra de las cuestiones que se observan cuando tratamos de la conformación de la agenda son las agendas referenciales. En bastantes ocasiones los participantes de los grupos de discusión señalan como temas de agenda cuestiones que no viven personalmente. Algunas veces sí se trata de problemas que viven algunos familiares cercanos, pero en otras ocasiones la agenda está dirigida por discursos dominantes referenciales que no tienen que ver con la realidad. Hemos observado esto para la inmigración, pero también para la vivienda, tal y como señalan Antón et al. (2007). La única excepción notable es la agricultura, pero ésta parece llevar un canal de negociación que no pasa por los medios de comunicación ni la agenda pública.

“En Castilla y León los que más saben (sobre las políticas de la Junta) son los agricultores y ganaderos, los que manejan la PAC, los que van al Junta, los sindicatos. Como esa gente es muy poderosa, hay mucha conexión entre la Junta y los sindicatos. Los lobbies están en la periferia, en el mundo rural, en las fábricas..." (GD1)

La última de las cuestiones que observamos es la falta de información del ciudadano con respecto a sus instituciones regionales, haciendo que no sepa juzgar a quién imputar las responsabilidades de la gestión. La Junta de Castilla y León no es muy conocida y la falta de medios de comunicación potentes de ámbito regional hace que no haya una identificación clara con los logros y desaciertos a la hora de imputar responsabilidades a los partidos políticos. Esta falta de información se suma a la falta de 
interés, lo cual favorece el fenómeno que hemos observado para Castilla y León de la adopción de agendas para su región que en realidad son réplicas de la agenda nacional, lo que lleva a una menor exigencia de cuentas ante los responsables políticos, haciendo que el eje nacional se superponga en el cálculo electoral de los ciudadanos.

"La Junta no tiene poder ni influencia. La NISSAN no la han conseguido conservar. Sin embargo, viene el País Vasco, por ejemplo, y propone generar industria aunque nos perjudique indirectamente y lo hace..." (GD1)

"No sé lo que ha hecho la Junta en estos 4 años (...). Yo me siento un poco desvinculada de la Junta: para mí la Junta sólo sé que me deniega la beca todos los años. Lo único que sé y que me llega el boletín oficial de la juventud de Castilla y León. Es lo único que sé de la Junta.” (GD1)

La influencia de los mass media para la agenda ciudadana en el caso de Castilla y León es relativa. Se percibe que los medios de comunicación autonómicos y locales reproducen un pensamiento monolítico que favorece un discurso y una opinión no crítica con el poder político en la Junta. Existe una barrera de comunicación que impide que la ciudadanía tenga acceso a información del territorio y sea crítica. Por otro lado, las redes sociales no parecen, hoy por hoy, una alternativa del todo viable al problema de la libertad informativa. Así lo reflejan en los grupos.

"Si, yo creo que los líderes son como viejos y no se interesan tanto por la comunicación, ni televisión, ni redes sociales y todo eso. No buscan imagen, como saben que el voto lo tienen ganado ¿para qué dar explicaciones?” (GD1)

"Somos la primera región en extensión en España y estamos como desaparecidos (en los medios de comunicación)...” (GD1)

En este contexto la ausencia de información propia y su bajo seguimiento por parte de los ciudadanos genera una mayor desafección. Los grandes medios de información crean opinión y discurso, pero no anclada en la realidad autonómica. Por su parte, las redes sociales suponen una atomización del espacio público y no generan discursos (opinión pública) sino relatos.

\section{Conclusiones}

El proceso de conformación de la agenda política se ha descrito como una interacción entre las agendas de los medios de comunicación y la agenda denominada "ciudadana". Cuando atendemos al nivel autonómico, observamos que ciudadanos y medios incorporan como parte de sus agendas cuestiones referenciales que no tienen un correlato directo con su entorno. Para el caso de Castilla y León, hemos visto que la agenda ciudadana está al margen de la agenda pública de su región, mimetizando 
la agenda nacional y conformando una situación de disgregación y distanciamiento que tiene un efecto directo en el desempeño percibido de la Comunidad Autónoma.

La baja conciencia regional parece ser el principal causante de este funcionamiento por parte de la ciudadanía de Castilla y León. Esta baja conciencia regional tiene un impacto directo en el modo en que se constituye la agenda ciudadana, que, incluso cuando se trata de cuestiones regionales, pasa a adoptar la agenda nacional. Con este artículo mostramos así que, para el estudio de las agendas, es preciso incorporar los distintos niveles de toma de decisión para poder entender la conformación de agendas. En este sentido, resulta relevante tanto el alto como el bajo grado de conciencia regional: en el caso de una conciencia regional baja hemos visto que las agendas nacionales pueden eclipsar las agendas regionales y locales, haciendo que la conformación de los temas más relevantes (o salient issues) no responda a la realidad de los ciudadanos, lo que llevará indefectiblemente a un mal desempeño percibido de las instituciones por parte de la ciudadanía, lo cual a su vez ahonda aún más en procesos de desafección con la autonomía y menor conciencia regional.

En el caso particular de Castilla y León observamos así que la mayoría de las cuestiones serían clasificadas, en la terminología que emplean Canel et al. (1996), como unobtrusive, es decir, como temas no directos en lo personal y que llegan al ciudadano solo mediatizados. En Castilla y León esto ocurre en mayor medida que el ámbito nacional, ya que se percibe que los medios de comunicación autonómico o local no son imparciales y no informan siempre de acontecimientos locales de cierto calado ya que se presupone un interés en ocultar información crítica para la Junta. Por consiguiente, observamos que, a pesar del poco reflejo en la bibliografía especializada, la cuestión de la conciencia local y regional (tanto su ausencia como su presencia) es un factor clave para poder entender el modo en que se configura la agenda de preferencias ciudadanas y en qué medida los medios de comunicación pueden afectarla o no y en qué ámbito. Por ello, en regiones con baja conciencia regional el poder de los medios aumenta a medida que hay menor relación de los individuos con su entorno inmediato, siendo su filtro mayor. Esto abre la posibilidad no sólo de entender los procesos que ocurren en la conformación de la agenda regional, sino que permite empezar a dar luz sobre cómo las agendas regionales pueden llegar a condicionar y fijar la agenda nacional en su caso.

\section{Bibliografía}

Antón, Fernando, Antonio Barroso, Eva Lidón, Elisa Rodríguez y Óliver Soto (2007): Vivienda y opinión pública en España, Centro de Investigaciones Sociológicas, Madrid. 
Bouza, Fermín (2012): "Las metodologías de agenda en el estudio de la opinión pública como base de la comunicación política" en Revista Digital de Marketing Aplicado, 8: 41-54. https://doi.org/10.17979/redma.2012.01.08.4731

BOUZA, Fermín y RODRÍGUEZ, Raquel (2004): "Los temas más destacados en la esfera pública española: elecciones 2004” publicado en el VII Congreso de Ciencia Política y de la Administración disponible en el siguiente enlace https://aecpa. es/files/congress/7/actas/area04/GT13/RODRIGUEZ-Raquel(URJC)yBOUZAFermin(UCM).pdf consultado el 16-07-2019.

Canel, María José, Llamas, Juan Pablo, y Rey-Lennon, Federico (1996): "El primer nivel del efecto agenda-setting en la información local: los" problemas más importantes" de la ciudad de Pamplona", en Universidad de Navarra, disponible en https://dadun.unav.edu/bitstream/10171/8581/1/20091117164551.pdf consultado el 15 de noviembre de 2019.

Cea D’Ancona, Ma Ángeles (2006): Metodología cuantitativa: estrategias y técnicas de investigación social. Madrid: Tecnos.

Cheng, Lifen, Juan José Igartua, Elena Palacios, Tania Acosta, José Antonio Otero,y Francisco Javier Frutos (2008): “Aversión vs. aceptación, dos caras de la misma moneda: un estudio empírico de encuadres noticiosos sobre inmigración en la prensa regional de Castilla y León" en ZER: Revista de Estudios de Comunicación= Komunikazio Ikasketen Aldizkaria, 14(26).

CIS (2015): Encuesta preelectoral del CIS de las elecciones autonómicas 2015. Comunidad Autónoma de Castilla y León. Estudio no 3094, junio de 2015.

CIS (2018): Barómetro de noviembre 2018. Estudio $n^{\circ} 3231$, noviembre de 2018.

CIS (2019): Encuesta preelectoral del CIS de las elecciones autonómicas 2019. Comunidad Autónoma de Castilla y León. Estudio no 3245, marzo-abril de 2019. COBB, Roger W. y Charles D. ELDER (1984): "Formación de la agenda", en Aguilar Villanueva, Luis F. (comp.): Problemas públicos y agenda de gobierno, México: Miguel Ángel Porrúa.

DAHL, Robert A. (1990): La poliarquía: participación y oposición. Madrid: Tecnos.

Dearing, James, y Everett M. Rogers (1988): “Agenda-setting research: Where has it been, where is it going" en Communication yearbook, 11: 555-594.

DEARING, James, y Everett M. ROGERS (1996): Communication Concepts 6: Agenda setting. Thousand Oaks: Sage Publications.

Doroshchuk, E., y Mantashashvili, M. (2016). "Interaction of processes of the news agenda setting in the russian regional media system (as exemplified by the republic of tatarstan)", en Academy Of Marketing Studies Journal, vol. 20, no especial 2: 58-63. 
DOWNS, Anthony (1972): "Up and down with ecology. The issue-attention cycle" en Public Interest, 28: 38-50.

De FLEUR, Melvin L. y Sandra BALL-ROKEACH (1975): Theories of Mass Communication. Nueva York: The Free Press.

FOOTE, N. N., \& HART, C. W. (1953): "Public opinion and collective behavior" en SHERIF, \& WILSON (Eds.), Group relations at the crossroads. Nueva York: Harper \& Brothers, pp. 308-331.

GIL CALVO, Enrique (2003): El miedo es el mensaje, Madrid, Alianza Ensayo.

GITTLIN, Todd (1979), "Prime time ideology: The hegemonic process in television entertainment" en Social Problems, n 26, pp. 251-266. https://doi. org/10.2307/800451

Gómez-Limón, José A., ignacio ATNACE y Margarita rico (2007). "Percepción pública del problema de la despoblación del medio rural en Castilla y León". Ager: Revista de estudios sobre despoblación y desarrollo rural, 6: 9-60.

Hernández Sánchez, Alfredo (2010): “Por qué en Castilla y León existe un bajo sentimiento regional a principios del siglo XXI? Una explicación sociológica” en Anales de Estudios Económicos y Empresariales, vol. XX, 2010, pp. 9-49.

KATZ, E. y Paul F. LAZARSFELD (1979): La influencia personal, Barcelona, HispanoEuropea.

Lijphart, Arend (1971): "Comparative politics and the comparative method" en American political science review. 65(3): 682-693.

McCOMBS, Maxwell E. (2006): Estableciendo la agenda: el impacto de los medios en la opinión pública y en el conocimiento. Barcelona: Paidós.

McCOMBS, Maxwell E. y Donald L. Shaw (1972): "The agenda-setting function of mass media” en Public opinion quarterly, 36(2): 176-187.

MCCOMBS, Maxwell E. y Donald L. SHAW. (1993): “The evolution of agendasetting research: Twenty-five years in the marketplace of ideas" en Journal of communication, 43(2): 58-67. https://doi.org/10.1111/j.1460-2466.1993. tb01262.x

MANIN, Bernand, Adam PRZEWORSKI y Susan C. STOKES (1999): Democracy, accountability and representation, Cambridge: Cambridge University Press.

Martínez-Sanz, Raquel, Patricia Durántez Stolle y Salomé Berrocal Gonzalo (2015): "La imagen de marca territorio de Castilla y León. Presencia y tratamiento en el periódico El Mundo" en Razón y Palabra, no 90.

MINISTERIO DE LA VIVIENDA (2004): Atlas Estadístico de las Áreas Urbanas en España 2004, Madrid, Secretaría General Técnica del Ministerio de Vivienda.

MORLINO, Leonardo (1996): "Las democracias" en PASQUINO, Gianfranco (comp.): Manual de Ciencia Política, Alianza Universidad, Madrid. 
RODRÍGUEZ DÍAZ, R. (2001): Los profesores universitarios como medios de comunicación: la "agenda-setting" de los alumnos y profesores, tesis doctoral, Universidad Complutense de Madrid, Madrid, disponible en http://www.ucm. es/BUCM/tesis/inf/ucm-t24877.pdf consultada el 17-07-2019.

RODRÍGUEZ DÍAZ, Raquel (2004): Teoría de la Agenda-Setting: aplicación a la enseñanza universitaria, Observatorio Europeo de Tendencias Sociales, Alicante. ROTH DEUBEL, André-Noël (2006): Políticas públicas. Formulación, implementación y evaluación. Bogotá: Aurora.

Rubio Ferreres, José María (2009): “Opinión pública y medios de comunicación. Teoría de lảagenda setting”, en Gazeta de Antropología, no 25/1, artículo 01.

SARTORI, Giovanni (2005): Elementos de teoría política, Madrid: Alianza Editorial.

Scheufele, Dietriam A., y David Tewksbury (2006): "Framing, agenda setting, and priming: The evolution of three media effects models" en Journal of communication, 57(1): 9-20. https://doi.org/10.1111/j.0021-9916.2007.00326.x

SOCYL (2016): Barómetro SOCYL Oleada Primavera 2016, Asociación Profesional de Sociología de Castilla y León, disponible en http://www.socyl.es/wp-content/ uploads/2017/01/Bar\%C3\%B3metro_Primavera16-.pdf consulta el 17-07-2019.

SOTO, Oliver (2015): El control sobre las Fuerzas Armadas: un estudio de la rendición de cuentas en las democracias (1960-2006), tesis doctoral, Universidad Complutense de Madrid, Madrid, disponible en https://eprints.ucm.es/37502/, consultada el 17-07-2019.

Spierkel, Karen (1981): Newspaper coverage of 1980 Quebec referendum a study of linguistic and regional agenda-setting in the politics of referenda, Tesis de máster inédita 2852, Universidad de Windsor.

TAMAYO, Manuel y Ernesto CARILLO (2005): "La formación de la agenda pública" en Foro Internacional, 45(4): 658-681.

Zucker, Harold G. (1978): “The Variable Nature of News Media Influence” en Brent D. R. (ed.): Communication Yearbook, NJ Tramaction Rooks: New Brunswiek. https://doi.org/10.1080/23808985.1978.11923728 
